\title{
Moisture curable toughened poly(lactide) utilizing vinyltrimethoxysilane based crosslinks
}

\author{
J. Schneider, K. Bourque, R. Narayan* \\ Michigan State University, Department of Chemical Engineering and Materials Science, MI 48823 East Lansing, USA
}

Received 17 February 2016; accepted in revised form 9 May 2016

\begin{abstract}
Vinyltrimethoxysilane (VTMOS) was grafted on to the backbone of poly(lactide) (PLA) through a free radical grafting reaction using reactive extrusion (REX) processing. The methoxy groups of the silane provide the modified PLA sites for crosslinking through a moisture induced pathway. VTMOS grafting efficiencies of up to $90 \%$ were obtained. The newly created methoxy functionality of the modified PLA readily undergoes hydrolysis and condensation forming siloxane crosslinks in the material. Crosslinking with VTMOS exhibited improved modulus, strength, and impact toughness while showing a decrease in ductility. Incorporating silanol-terminated poly(dimethylsiloxane) (OH-PDMS) resulted in the formation of longer siloxane crosslinks. These samples showed an increase in modulus and impact toughness due to the crosslinking, while the longer siloxane linkages resulted in improved ductility and tensile toughness. This is unusual for polymers toughened through crosslinking reactions. Scanning Electron Microscopy (SEM) of the fractured surfaces showed the presence of these elongated siloxane crosslinks. This enhanced ability for the modified PLA to deform and absorb energy results in the increase in both impact and tensile toughness.
\end{abstract}

Keywords: biodegradable polymers, reactive extrusion, free radical grafting, moisture induced crosslinking, PLA

\section{Introduction}

Poly(lactide) (PLA) is receiving much attention in the polymer materials space because it is produced from renewable resources and is biodegradable/compostable. Thanks to the commercialization processing improvements of PLA producing companies like NatureWorks, LLC, its cost is around $\$ 1 / \mathrm{lb}$, making it an intriguing cost-competitive alternative to traditional petroleum based plastics [1]. However, PLA possesses certain inherent polymer properties such as low impact toughness [2], poor heat [3] and hydrolytic stability [4], and weak melt strength [5] that have prevented it from more wide scale commercial use.

Compared to poly(styrene), PLA has very similar mechanical properties such as tensile strength and modulus, as well as similar brittleness and low impact strength [6]. These limitations of poly(styrene) led to the development of modified high-impact polymers and copolymers (ex. ABS, HIPS) to further its commercial viability. Now in recent years, the toughening of PLA has undergone the same level of interest in effort to develop a toughened PLA. The typical methods of this include plasticization, copolymerization, and melt blending.

Some research has been done utilizing vinyl functionality to graft reactive groups onto PLA's backbone, making it more susceptible to modification. The introduction of new functional groups onto PLA allows for the creation of new materials, with potentially increased properties due to the incorporation of new reactive chemistries. While many different functionalities have been grafted onto PLA, including acrylic acid [7] and acrylamides [8], the most popular method of PLA melt grafting is with maleic an- 
hydride. This grafting is typically done using reactive extrusion (REX) processing making it cost-competitive and commercially scalable. Maleic anhydride grafted PLA polymers (MA- $g$-PLA) have been used as compatibilizers for native [9] and thermoplastic starches [10] where they have promoted strong interfacial adhesion and better dispersion for these biocomposites. Other researchers have used MA- $g$ PLA as an reactive intermediary to graft more flexible compounds such as poly(ethylene glycol) [11] and citrate [12] onto PLA to increase its toughness.

PLA can also be toughened by introducing an appropriate level of crosslinking into the material. The two common procedures to crosslink thermoplastic polymers are copolymerization with a multifunctional monomer and introducing reactive compounds onto the polymer backbone. Such reactive compounds include the vinyl functionalities previously described, followed by additional crosslinking steps. Crosslinking through the grafting of a reactive compound route has been commonly done with triallyl isocyanurate (TAIC). This has been performed both in the melt via free radical initiation [13] and crosslinking prefabricated samples via radiation curing [14]. TAIC is a highly reactive material making it difficult to control the extent of grafting. And while crosslinking is an effective method of increasing impact toughness, it typically results in a loss in ductility and therefore a decrease in tensile toughness.

Vinylalkoxysilane grafting appears to be an excellent combination of the two methods. The vinyl functionality can be easily incorporated onto the PLA backbone via free radical grafting in the melt phase, while the alkoxysilane functionality is capable of forming crosslinks through moisture curing. This procedure was first introduced by Dow Corning for the crosslinking of polyolefins and termed the SioPlas method [15]. This two-step method of free radical grafting followed by moisture cured crosslinking increases the chemical and thermal resistance of the polyolefins, while also increasing the crack propagation and impact resistance [16]. The advantages of this crosslinking method compared to other common techniques such as initiation by high energy radiation and peroxides are energy saving, higher productivity, and the crosslinked products contain stable siloxane ( $\mathrm{Si}-\mathrm{O}-\mathrm{Si}$ ) bonds [17]. However, this method still does result in a loss in ductility. Han et al. [18] reported a study adopting this SioPlas method to PLA in which an improvement in thermal stability, hydrolysis resistance, and strength/stiffness properties occurred. The crosslinked PLA showed an increase in tensile strength and modulus along with a decrease in ductility/elongation, similar to SioPlas. In addition, they only achieved very low grafting efficiency (max of $37 \%$ ). This study used a hot water crosslinking step, which Rahmat et al. [19] recently showed leads to excessive hydrolytic degradation of PLA. At long crosslinking times, hydrolytic degradation becomes the dominant mechanism leading to network defects, causing loose gel structures.

In this paper, improvements to the REX processing are discussed in detail and their effects on the grafting efficiency of VTMOS onto PLA are reported. The improvements result in drastically better grafting efficiency and therefore less VTMOS is needed. After process optimization, a silanol-terminated poly (dimethylsiloxane) (OH-PDMS) was added into the system to aid in the crosslinking. This resulted in longer and more flexible crosslinks being formed which had a large positive impact on mechanical properties. Crosslinking was done without the previously used hot water bath to prevent excessive hydrolytic degradation. Tensile and impact properties where determined and the siloxane crosslinking effect was examined.

\section{Experimental}

\subsection{Materials}

PLA resin pellets (3051D) with a weight average molecular weight of $150000 \mathrm{~g} / \mathrm{mol}(\mathrm{PDI}=1.7)$ and $8 \%$ meso-lactide content $\left(T_{\mathrm{m}}=\sim 155^{\circ} \mathrm{C}\right)$ were purchased from NatureWorks LLC (NE, USA). Vinyltrimethoxysilane (VTMOS, $b p=123{ }^{\circ} \mathrm{C}$ ) and 2,5Bis(tert-butylperoxy)-2,5-dimethylhexane (Luperox 101) were obtained from Sigma-Aldrich (WI, USA). The silanol terminated polydimethylsiloxane $(\mathrm{OH}-$ PDMS) was from Gelest and possessed a molecular weight of $\sim 550 \mathrm{~g} / \mathrm{mol}$.

\subsection{Compounding and sample preparation}

The PLA resin pellets were dried for 24 hours in a $70^{\circ} \mathrm{C}$ oven to remove moisture. This is crucial for PLA in extrusion processes because it has a tendency to hydrolytically degrade as a result of back-biting and intermolecular transesterification reactions [20]. The 
VTMOS grafting process was carried out using a ZSK $30 \mathrm{~mm}$ co-rotating twin screw extruder (Werner Pfleiderer, NJ, USA) with an $L / D$ ratio of 30 . The dried PLA pellets were premixed with the required amounts of VTMOS, OH-PMDS, and Luperox before being fed into the hopper using a gravimetric feeder. All samples were compounded at a throughput of $6 \mathrm{~kg} / \mathrm{hr}$, cooled in a water bath, and then pelletized. The pellets were tumbled in a bin with absorbent towels to remove the surface moisture. Some moisture was retained in the sample to promote hydrolysis of the methoxy groups and promote the crosslinking chemistry during downstream processing. The screw speed and temperature profile for all compounding were $125 \mathrm{rpm}$ and 120, 140, 160, 170, $170,165^{\circ} \mathrm{C}$, respectively. It is necessary for the first temperature zone to be $120^{\circ} \mathrm{C}$ or lower to prevent flash evaporation of the VTMOS in the hopper. Previously, Han et al. [18] used an initial feed zone temperature of $170^{\circ} \mathrm{C}$ and noted very low grafting, even though large percentages were used. This could be because a large amount of it did not even enter the extruder due to flash evaporation as soon as it entered the hopper. This same study used a screw speed of $60 \mathrm{rpm}$ which is rather slow and results in poor mixing. Poor mixing leads to slower reactions, which is another potential reason for the low grafting percentages.

Tensile and impact test specimens were compression molded using a Carver Laboratory Press (IN, USA), immediately after tumble-drying of the pellets. Immediate processing was done to ensure that the crosslinking reaction had not started prior to sample fabrication. The platen were heated to $180^{\circ} \mathrm{C}$ and the filled mold was pressed at a minimum of 10 ton for 3 minutes. Cooling water was then turned on to cool the platen. The mold was removed when the temperature reached below $40^{\circ} \mathrm{C}$, or approximately $10 \mathrm{~min}$ utes. After removal from the mold, the specimens were placed into a $50^{\circ} \mathrm{C}$ oven for 3 days to pull off any residual water and subsequently drive the condensation reaction forward to form the siloxane crosslinks. In the past, this crosslinking step was done by placing the samples in hot water for up to 20 hours, which led to significant degradation of the PLA [19]. Test specimens were stored in a zip-top bag with as much air removed as possible and were allowed to rest for 2 weeks prior to mechanical testing.

\subsection{Percent grafting determination}

The grafting percent of VTMOS onto PLA was measured immediately after compounding and then again prior to mechanical testing using two different techniques, TGA and proton NMR. In the TGA method, the amount of free or ungrafted VTMOS can be determined by isothermally holding the sample above the boiling point of VTMOS $\left(123^{\circ} \mathrm{C}\right)$, for 20 minutes. At this temperature, the free VTMOS evaporates and the weight loss represents the un-grafted VTMOS. Also at this temperature, PLA begins to slightly decompose as seen by a gradual negative slope in the weight percent versus time graph. To correct for this, the slope of the line in the 18-20 minute range is extrapolated back to time zero and is used for determining the percent grafting. The slope is calculated at the end of the isotherm since all free VTMOS has been removed and a steady slope is reached. The extrapolated time zero weight $\%$ is the grafted VTMOS and PLA percentage in the sample. Therefore the weight $\%$ difference from the extrapolated and starting values, is due to the loss of the ungrafted VTMOS.

Proton NMR was also used to establish percent grafting. The ratio of the vinyl to methoxy proton integrations from neat VTMOS was compared to that of the reacted material. Neat VTMOS shows an integration ratio of 1:3 for vinyl to methoxy protons. So as the VTMOS grafts onto PLA through the reaction of the vinyl group, the signal corresponding to this vinyl group decreases. This results in a decrease in the vinyl to methoxy proton integration ratio. Therefore percent grafting can be calculated with NMR by Equation (1):

Grafting $[\%]=\frac{[v: m]_{\text {neat }}-[v: m]_{\text {exp }}}{[v: m]_{\text {neat }}} \cdot 100$

where $[v: m]_{\text {neat }}$ and $[v: m]_{\exp }$ are the integration ratios corresponding to the vinyl and methoxy groups of VTMOS for the pure/neat material and the experimentally reacted materials, respectively. For the pure material, this in theory should be 1:3, however in practice it is typically slightly higher due to the hydrolysis of some methoxy groups.

NMR was primarily used to verify the grafting percentage determinations obtained by TGA. The grafting percentages calculated by TGA and NMR showed less than 5\% statistical variation. Therefore it was con- 
cluded that TGA can accurately determine VTMOS grafting efficiency, and was then used as the primary method to determine grafting. This TGA/NMR method for experimentally determining VTMOS grafting efficiency has been used to monitor/determine VTMOS grafting onto soybean oil [21].

\subsection{Mechanical testing}

The tensile properties of the siloxane crosslinked samples were tested using a United Testing Systems (CA, USA) SFM-20 load frame with a $1000 \mathrm{lb}(4448 \mathrm{~N})$ load cell. The dog-bone shaped specimens had a 2 inch gage length and cross-sectional dimensions of $0.5 \times 0.125$ inches $(12.7 \times 3.175 \mathrm{~mm})$. The testing rate used was $0.2 \mathrm{inch} / \mathrm{min}(5.08 \mathrm{~mm} / \mathrm{min})$, which resulted in most failures occurring between $30-120 \mathrm{sec}$ onds. A minimum of 5 replicates were tested for each compound.

The notched IZOD impact properties were determined using a TMI pendulum impact tester (Testing Machines, Inc., DE, USA), following ASTM D256 utilizing a $1 \mathrm{ft}-1 \mathrm{~b}(0.113 \mathrm{Nm})$ pendulum. The specimens had cross-sectional dimensions of $0.5 \times 0.125$ $(12.7 \times 3.175 \mathrm{~mm})$ inches, and the notch was 0.1 $(0.254 \mathrm{~mm})$ inch deep. After notching, the specimens were allowed to rest at least 24 hours prior to impact testing. A minimum of 5 replicates were impact tested for each sample set.

\subsection{Morphology (SEM)}

The morphologies of the siloxane crosslinked samples were studied using a JEOL 6610V (Japan Electron Optics Laboratories, Japan) Scanning Electron Microscope (SEM). The fractured surfaces following tensile and impact testing were analyzed after coating with osmium.

\section{Results and discussion}

\subsection{Determination of VTMOS grafting}

The previous study by Han et al. [18] showed that VTMOS can be grafted onto the backbone of PLA using a free radical initiator through reactive extrusion (REX) processing. However, with the processing conditions used, very low grafting percentages resulted. Due to this low grafting efficiency, these researchers used high concentrations of VTMOS, up to $8 \mathrm{wt} \%$. This VTMOS concentration results in a molar ratio of $\sim 70$ VTMOS per PLA chain (based on a num- ber average molecular weight of $120000 \mathrm{~g} / \mathrm{mol}$ ), which is excessive and could lead to an extremely high crosslink density as well as a dramatic increase in the cost of the final material. Therefore, the initial portion of this study was to optimize the process to give enhanced grafting efficiency for our materials while using lower concentrations of VTMOS, and thereby reducing costs.

In attempt to increase the grafting efficiency, a lower VTMOS concentration ( 0.5 and $1.0 \mathrm{wt} \%$ ), a lower feed zone temperature (starting at $120^{\circ} \mathrm{C}$ - so no VTMOS loss due to evaporation), and higher screw speed $(125 \mathrm{rpm})$ were used. Initially a higher free radical initiator (Luperox) concentration was also used $(0.5 \mathrm{wt} \%)$, however this resulted in a rigid and highly brittle thermoset material (incapable of dissolving in solvent). This was caused by an unwanted additional reaction of $\mathrm{Si}-\mathrm{O}-\mathrm{C}$ linkages formed through the methoxy groups and PLA during extrusion. This two sided reaction resulted in the formation of the thermoset. Reducing the Luperox concentration to 0.25 and $0.1 \mathrm{wt} \%$ yielded products with slightly lower grafting efficiencies of 87.49 and $82.91 \%$, respectively. However, this reaction was without the unwanted $\mathrm{Si}-\mathrm{O}-\mathrm{C}$ linkages responsible for the formation rigid thermoset. Both samples were fully dissolvable in solvent, indicating that no crosslinking occurred through REX, but rather the grafting was done solely via the vinyl attachment onto PLA.

The mechanism for the vinyl attachment of VTMOS onto the PLA backbone is shown in Figure 1. This mechanism for melt free radical grafting utilizing vinyl functionality is well accepted and has been used to incorporate many different chemical species onto PLA. The first step is the thermal decomposition of a free radical initiator (Luperox) resulting in the production of radicals. The generated radical abstracts hydrogen from the $\alpha$-carbon, creating radicals on the PLA chain. This hydrogen abstraction of PLA was first shown by Avella et al. [22] in the reaction with butyl acrylate. The PLA radicals are then capable of coupling with radicals on the vinyl group of VTMOS, as shown below. A potential side reaction involves the PLA radicals coupling with another material, the initiator radical, hydrogen, or another polymer radical, potentially leading to an unwanted result such as $\beta$-scission, which is noted by a molecular weight 
<smiles>C=C[Si](O)(O[Na])O[Na]</smiles>

VTMOS

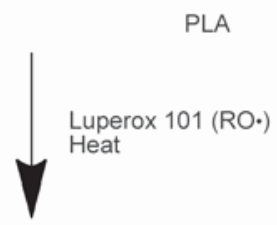<smiles>COC(=O)C(C)OC(=O)C(C)(CC[Si]([O-])(O)O[Na])OC(=O)C(C)OC(=O)C(C)C</smiles>

Figure 1. A schematic mechanism for the free radical initiated grafting of VTMOS on PLA

decrease in processing. The vinyl grafting efficiency was determined by NMR using the decrease in the vinyl to methoxy proton integration ratio as well as a slight change in the ratio of the PLA peaks corresponding to the methyl hydrogens and the hydrogen on the C-backbone. These two components prove that VTMOS is grafted onto the PLA backbone as described in Figure 1.

Following VTMOS grafting, a moisture induced crosslinking of the grafted polymers can occur. First the methoxy groups of VTMOS readily hydrolyze in the presence of water, forming silanol functionality ( $\mathrm{Si}-\mathrm{OH})$. The silanols condense to form siloxane linkages leading to the formation of crosslinked compounds. These short siloxane linkages from the coupling of two VTMOS molecules is not flexible enough to impart any toughening benefit. In fact, just crosslinking PLA with VTMOS yields a material with less ductility. Therefore, in effort to lengthen the siloxane linkages and create a true toughening effect, a silanol functionalized PDMS (OH-PDMS) was incorporated to take part in the condensation portion of the crosslinking reaction. This hydrolysis and condensation crosslinking reaction is shown in Figure 2.

After fabrication of test specimens and the following moisture cure, samples were again analyzed to determine grafting efficiency and for the formation of gels. Over this crosslinking period, the compounds expe- rienced very little change in grafting of VTMOS and some samples showed a slight increase. This increase in grafting can be explained by some of the free, or unreacted VTMOS being incorporated in the crosslinking reaction thereby becoming part of the crosslinked material. A few of the samples showed a decrease in grafting which can be attributed to the weight loss of methanol during testing, which is created through the hydrolysis step. VTMOS can also migrate to the surface and evaporate instead of crosslinking. The grafting percentages for the compounds after REX and after the crosslinking period are shown in Table 1. For the VTMOS only samples, the higher Luperox concentration results in slightly higher grafting. With the addition of OH-PDMS, the opposite trend occurs where $0.1 \%$ Luperox results in higher grafting. The other trend to notice is that there was lower grafting efficiency for the $0.5 \mathrm{wt} \%$ VTMOS samples compared to the $1 \mathrm{wt} \%$ VTMOS samples. This is probably due to small losses of VTMOS in processing due to evaporation, where when using less material, the small losses result in larger percentages.

After the moisture curing step, the compounds were again placed in dichloromethane to dissolve. All of the samples experienced gel-swell rather than dissolving. This indicates that the siloxane crosslinking had in fact occurred through the hydrolysis and con- 
2<smiles>COC(=O)C(C)OC(=O)C(C)(CC[Si](C)(O)O[Na])OC(=O)C(C)OC(=O)C(C)C</smiles><smiles>CC(C)C(=O)OC(C)C(=O)OC(C)(CC[Si](O)(O)O)C(=O)OC(C)C(=O)OCCO</smiles>

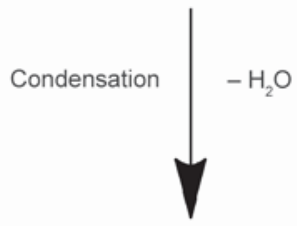<smiles>CCCCCC(C)OC(=O)C(C)OC(=O)C(C)OC(=O)C(C)OC(=O)C(C)OC(=O)C(C)OC(=O)C(C)OC(=O)C(C)OC(=O)C(C)OC(=O)C(C)OC(=O)C(C)OC(C)(C)O[Si](C)(C)O[Si](O)(O)CC[Si](O)(O)OC</smiles>

Figure 2. A mechanism showing the hydrolysis of the methoxy groups and the condensation resulting in siloxane crosslinked PLA

densation steps shown. The REX procedure used was shown to be effective in its ability to enhance VTMOS grafting efficiency onto PLA compared to previous reports.

\subsection{Mechanical properties}

The tensile properties of neat and REX modified PLA samples are shown in Table 2. The yield stress $\left(\sigma_{\mathrm{y}}\right)$ was taken as the maximum stress value achieved 
Table 1. Determination of percent VTMOS grafting post REX processing and post two week crosslinking period

\begin{tabular}{|c|c|c|c|c|}
\hline \% VTMOS & \% OH-PDMS & \% Luperox & \% Graft.intial & \% Graft.2 weeks \\
\hline 0.5 & 1 & 0.10 & 78.2 & 70.5 \\
\hline 0.5 & 1 & 0.25 & 66.1 & 75.1 \\
\hline 0.5 & 3 & 0.10 & 63.5 & 70.3 \\
\hline 0.5 & 3 & 0.25 & 63.7 & 83.1 \\
\hline 1.0 & 1 & 0.10 & 89.5 & 86.5 \\
\hline 1.0 & 1 & 0.25 & 86.4 & 77.8 \\
\hline 1.0 & 3 & 0.10 & 79.3 & 79.8 \\
\hline 1.0 & 3 & 0.25 & 76.4 & 89.9 \\
\hline 1.0 & 0 & 0.10 & 82.9 & 87.4 \\
\hline
\end{tabular}

for the specimens and the elongation at fracture $\left(\varepsilon_{\mathrm{f}}\right)$ is the strain at break. The modulus $(E)$ of the specimens was determined by measuring the slope of the stress-strain curve in the linear elastic region, within the first $0.5 \%$ strain. It should first be mentioned that the mechanical properties experimentally determined for neat PLA are comparable to the properties reported by NatureWorks in their technical data sheet. As mentioned earlier, crosslinking PLA with only VTMOS results in a decrease in ductility. This was observed in our results as the test specimens showed an increased modulus coupled with a loss in elongation. This is due to the formation of a crosslinked network where the crosslinks are short, and rather stiff in nature. As OH-PDMS is added and incorporates in the crosslinking, the siloxane crosslinks become longer and much more flexible due to the physical characteristics of this bond. The short siloxane linkages formed by the coupling of VTMOS does not impart flexibility like the longer OH-PDMS chains. With an increase in OH-PDMS content, an increase in elongation is experienced along with a slight reduction in yield stress. In fact, the VTMOS only specimens did not show a true yield stress but rather an increase in stress until fracture, and the neat PLA fractured immediately following yielding. When $\mathrm{OH}-$ PDMS is incorporated, a true yielding is experienced and examples of such samples are shown in Figure 3. However, it is possible that some of the increase in elongation observed for the samples with OH-PDMS is due to a lubricating effect from un-reacted $\mathrm{OH}-$ PDMS.

From Figure 3 it is clear to see that by incorporating OH-PDMS, much higher elongations to failure are experienced. However, the very unique result from this modification is that the elongation was increased
Table 2. Tensile properties of the siloxane crosslinked PLA samples

\begin{tabular}{|c|c|c|c|c|c|}
\hline \% VTMOS & \% OH-PDMS & \% Luperox & $\begin{array}{c}\boldsymbol{E} \\
\text { [GPa] }\end{array}$ & $\begin{array}{c}\sigma_{\mathbf{y}} \\
{[\mathbf{M P a}]}\end{array}$ & $\begin{array}{c}\boldsymbol{\varepsilon}_{\mathrm{f}} \\
{[\%]}\end{array}$ \\
\hline 0.5 & 1 & 0.10 & 3.40 & 54.68 & 53.07 \\
\hline 0.5 & 1 & 0.25 & 3.25 & 55.02 & 5.94 \\
\hline 0.5 & 3 & 0.10 & 3.67 & 44.95 & 9.97 \\
\hline 0.5 & 3 & 0.25 & 3.07 & 45.51 & 4.45 \\
\hline 1.0 & 1 & 0.10 & 3.43 & 55.36 & 3.59 \\
\hline 1.0 & 1 & 0.25 & 3.33 & 53.16 & 4.05 \\
\hline 1.0 & 3 & 0.10 & 3.71 & 45.44 & 3.24 \\
\hline 1.0 & 3 & 0.25 & 3.48 & 45.30 & 4.49 \\
\hline 1.0 & 0 & 0.10 & 3.73 & 58.67 & 2.40 \\
\hline 1.0 & 0 & 0.25 & 3.46 & 55.92 & 1.92 \\
\hline 0 & 0 & 0 & 2.62 & 58.67 & 2.93 \\
\hline
\end{tabular}

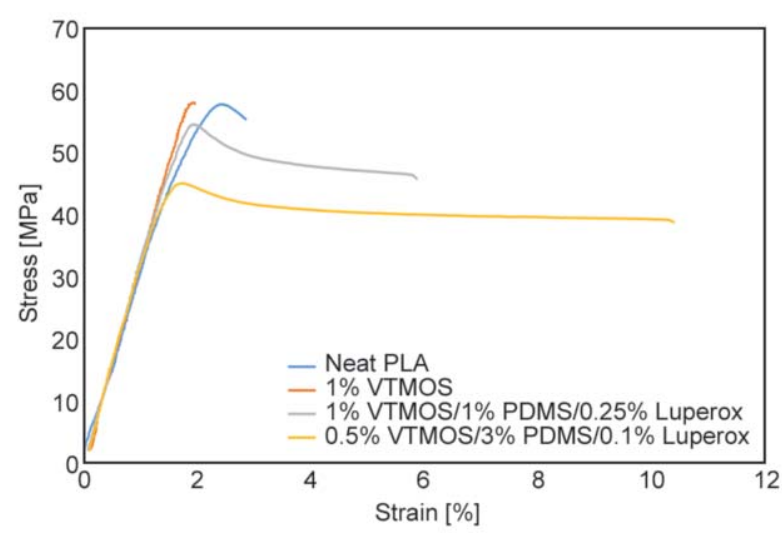

Figure 3. Representative stress-strain curves of neat PLA, VTMOS crosslinked PLA, and crosslinked samples incorporating 1 and 3\% OH-PDMS

without reduction in moduli of the specimens. In fact, the crosslinked samples with OH-PDMS showed a significantly higher modulus than neat PLA. This is caused by crosslinking a stiff material with a very ductile polymer. The crosslinked structure initially provides rigidity, raising the modulus. Yet these ductile crosslinks are capable of stretching under the applied load until they reach their limit, where the PLA then fails. This explains why even with increased 
elongation, a brittle failure was still observed. All tensile specimens showed a flat and smooth fracture surface, indicating a brittle fracture. The OH-PDMS crosslinked samples are both stiffer and more ductile in comparison to neat PLA, resulting a significant increase in the tensile toughness, as seen by the area under the curves. The addition of OH-PDMS does result in a slight reduction in ultimate stress, but with the increases in elongation and modulus, an increase in toughness is still experienced.

Another thing of note is how the crosslink density affects the tensile properties. Assuming $80 \%$ grafting efficiency, 0.5 and $1 \mathrm{wt} \%$ of VTMOS results in $~ 4$ and $\sim 8$ VTMOS groups grafting onto each PLA chain, respectively. Therefore these samples experience different crosslink densities. The lower crosslink density samples $(0.5 \mathrm{wt} \%$ VTMOS) show higher elongations to failure, while the high crosslink density samples have a higher modulus. This fits with theory and is what would be expected when dealing with crosslinked samples. The higher amount of crosslinks in a specific area result in the material not being able to stretch and deform as much, coincidentally resulting in a higher modulus. However, regardless of the VTMOS content in the samples, a significant increase in tensile toughness was observed with the presence of OH-PDMS due to increases in both elongation to failure and modulus compared to neat PLA.

IZOD Impact testing also showed a significant increase in toughness $(\sim 25 \%)$ for the modified samples. However there was no discernible trend based on VTMOS, OH-PDMS, or Luperox concentrations, as seen in Table 3. It appears that with such a dynamic and fast fracture, the extent of crosslinking (VTMOS concentration) and the crosslink length (OH-PDMS concentration) has no direct effect. But rather, a siloxane crosslinked sample of any nature shows approximately a $25 \%$ increase in impact toughness over neat PLA. The quick fracture likely doesn't allow for the longer siloxane linkages to stretch and absorb more energy, yielding a higher toughness as is seen with higher OH-PDMS concentrations in tensile testing. But rather the presence of these siloxane crosslinks is enough to increase the impact toughness compared to neat PLA.

\subsection{Morphology}

The tested samples were analyzed using SEM to determine if the siloxane crosslinks affected the morphology of the fractured surfaces. Figure 4 shows the fractured surfaces of select samples following tensile testing. The neat PLA sample in Figure 4 a shows an extremely rough surface in comparison to the others which are just VTMOS crosslinked (Figure $4 \mathrm{~b}$ ) and containing both VTMOS and OH-PDMS crosslinking (Figure 4c). This extremely rough surface corresponds to the brittle fracture experienced by the neat PLA samples during testing. Not only do the siloxane crosslinked samples exhibit a smoother fracture surface but they show siloxane pullout. In these samples, small holes (black circles) of where the siloxane was pulled out are visible along with the analogous opposite, the stretched siloxane (white circles). The sample containing OH-PDMS shows the presence of long white strands which are most likely the long siloxane linkages from the PDMS polymers.

The morphologies of the impact test samples are shown in Figure 5. All of the fractured surfaces exhibited a rippled pattern morphology, seen by the

Table 3. Notched IZOD impact toughness of siloxane crosslinked samples compared to neat PLA

\begin{tabular}{|c|c|c|c|c|c|}
\hline$\%$ VTMOS & $\%$ OH-PDMS & \% Luperox & $\begin{array}{c}\text { Impact energy } \\
{[\mathrm{J} / \mathrm{m}]}\end{array}$ & Standard deviation & $\%$ Increase vs neat PLA \\
\hline 0.5 & 1 & 0.10 & 26.71 & 0.35 & 22.6 \\
\hline 0.5 & 1 & 0.25 & 28.36 & 0.29 & 30.2 \\
\hline 0.5 & 3 & 0.10 & 27.14 & 0.66 & 24.6 \\
\hline 0.5 & 3 & 0.25 & 27.30 & 0.44 & 25.3 \\
\hline 1.0 & 1 & 0.10 & 27.51 & 1.05 & 26.3 \\
\hline 1.0 & 1 & 0.25 & 27.51 & 0.61 & 26.3 \\
\hline 1.0 & 3 & 0.10 & 27.45 & 0.08 & 26.0 \\
\hline 1.0 & 3 & 0.25 & 25.39 & 0.50 & 16.5 \\
\hline 1.0 & 0 & 0.10 & 26.71 & 0.25 & 22.6 \\
\hline 1.0 & 0 & 0.25 & 27.83 & 0.36 & 27.7 \\
\hline 0 & 0 & 0 & 21.78 & 2.86 & 0 \\
\hline
\end{tabular}




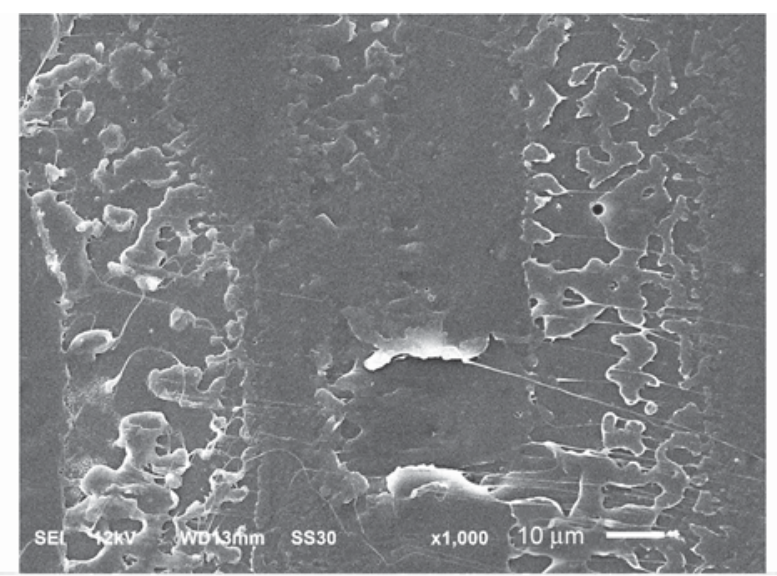

a)

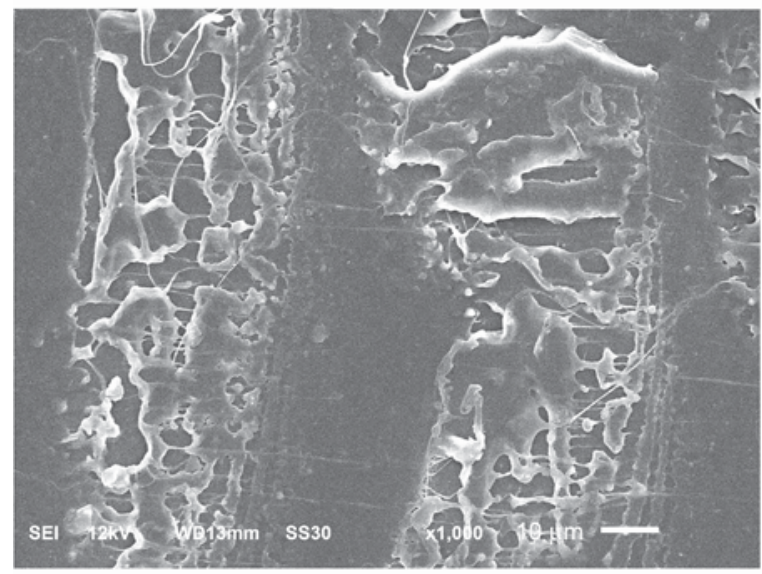

b)

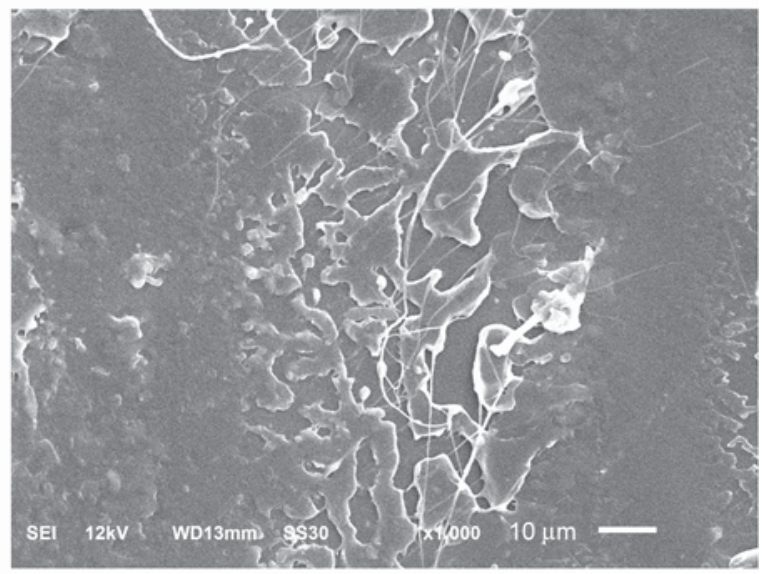

c)

Figure 4. SEM micrographs of the tensile fractured surfaces of a) neat PLA, b) $1 \%$ VTMOS - 0.25\% Luperox, and c) $0.5 \%$ VTMOS - 3\% OH-PDMS - 0.1\% Luperox samples

vertical columns in the figure. The crosslinked samples show more visual connectivity of the raised sections of the surface, which are from the crosslinking. For the sample with OH-PDMS, the same long linkages are visible as seen in the tensile samples, due to the longer siloxane crosslinks. This added connectivity and ability to deform prior to fracture is the cause of the increase in impact energy.

\section{Conclusions}

VTMOS was grafted onto PLA using REX processing and moisture cured to form a siloxane crosslinked PLA material. Process improvements compared to a previous study resulted in greater than a two fold increase in grafting efficiency of VTMOS onto the PLA backbone. This resulted in less VTMOS used, lowering the cost of the final product. $\mathrm{OH}-$ PDMS was added to lengthen the siloxane crosslinks, resulting in improved tensile toughness. The samples crosslinked with only VTMOS showed improvements in modulus, strength, and impact prop- erties with a decrease in ductility, as previously reported. However, by incorporating OH-PDMS into the crosslinking reaction, not only did an increase in modulus and impact toughness occur compared to neat PLA, but increased elongation to failure was observed resulting in the improvement in tensile toughness. SEM of the fractured surfaces showed the presence of the stretched siloxane crosslinks for the impact samples and siloxane pullout for the tensile samples. This enhanced ability for the material to deform and absorb energy gives reason for the increase in toughness.

\section{References}

[1] Auras R., Harte B., Selke S.: An overview of polylactides as packaging materials. Macromolecular Bioscience, 4, 835-864 (2004).

DOI: $10.1002 / \mathrm{mabi} .200400043$

[2] Jacobsen S., Fritz H. G.: Plasticizing polylactide - The effect of different plasticizers on the mechanical properties. Polymer Engineering and Science, 39, 13031310 (1999). DOI: $10.1002 /$ pen.11517 


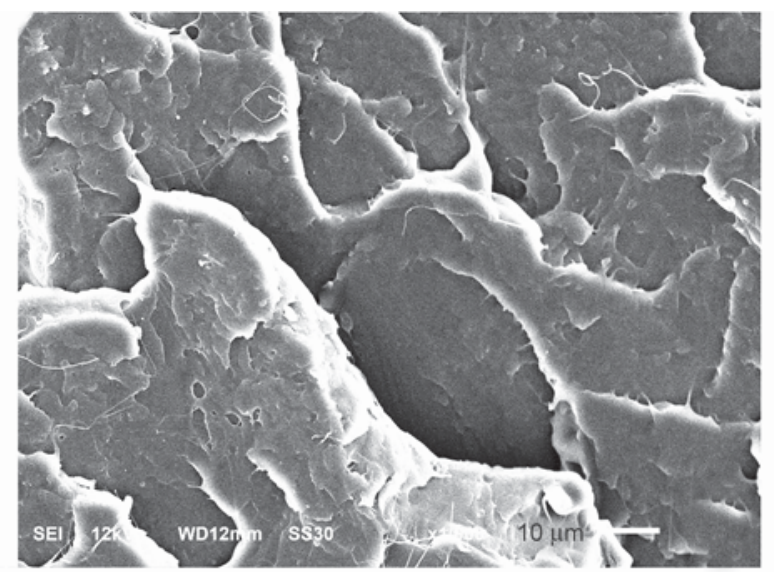

a)

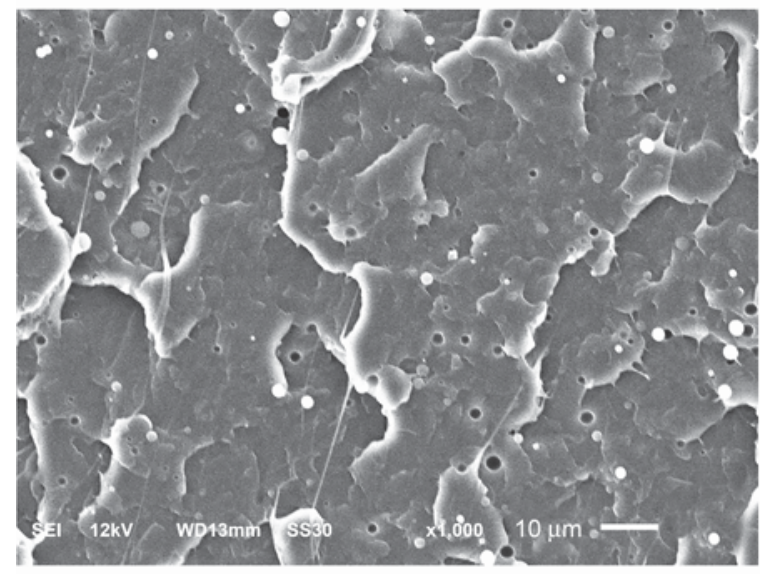

b)

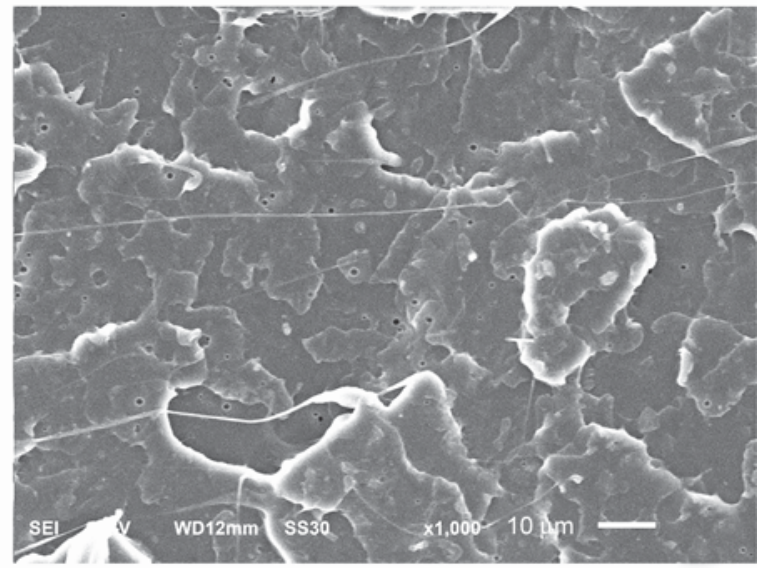

c)

Figure 5. SEM micrographs of the impact test fracture surfaces of a) neat PLA, b) $1 \%$ VTMOS $-0.25 \%$ Luperox, and c) $1 \%$ VTMOS - 1\% OH-PDMS - 0.1\% Luperox samples

[3] Jamshidi K., Hyon S-H., Ikada Y.: Thermal characterization of polylactides. Polymer, 29, 2229-2234 (1988). DOI: 10.1016/0032-3861(88)90116-4

[4] De Paula E. L., Mano V., Pereira F. V.: Influence of cellulose nanowhiskers on the hydrolytic degradation behavior of poly(D,L-lactide). Polymer Degradation and Stability, 96, 1631-1638 (2011).

DOI: $10.1016 /$ j.polymdegradstab.2011.06.006

[5] Dorgan J. R., Janzen J., Clayton M. P., Hait S. B., Knauss D. M.: Melt rheology of variable L-content poly (lactic acid). Journal of Rheology, 49, 607-619 (2005). DOI: $10.1122 / 1.1896957$

[6] Raquez J-M., Habibi Y., Murariu M., Dubois P.: Polylactide (PLA)-based nanocomposites. Progress in Polymer Science, 38, 1504-1542 (2013).

DOI: 10.1016/j.progpolymsci.2013.05.014

[7] Wu C-S.: Improving polylactide/starch biocomposites by grafting polylactide with acrylic acid - Characterization and biodegradability assessment. Macromolecular Bioscience, 5, 352-361 (2005).

DOI: $10.1002 / \mathrm{mabi} .200400159$

[8] Zare A., Morshed M., Bagheri R., Karimi K.: Effect of various parameters on the chemical grafting of amide monomers to poly(lactic acid). Fibers and Polymers, 14, 1783-1793 (2013).

DOI: $10.1007 / \mathrm{s} 12221-013-1783-\mathrm{y}$
[9] Carlson D., Nie L., Narayan R., Dubois P.: Maleation of polylactide (PLA) by reactive extrusion. Journal of Applied Polymer Science, 72, 477-485 (1999).

DOI: 10.1002/(SICI)1097-4628(19990425)72:4<477::

$$
\text { AID-APP3>3.0.CO;2-Q }
$$

[10] Huneault M. A., Li H.: Morphology and properties of compatibilized polylactide/thermoplastic starch blends. Polymer, 48, 270-280 (2007).

DOI: $10.1016 /$ j.polymer.2006.11.023

[11] Hassouna F., Raquez J-M., Addiego F., Dubois P., Toniazzo V., Ruch D.: New approach on the development of plasticized polylactide (PLA): Grafting of poly(ethylene glycol) (PEG) via reactive extrusion. European Polymer Journal, 47, 2134-2144 (2011).

DOI: $10.1016 /$ j.eurpolymj.2011.08.001

[12] Hassouna F., Raquez J-M., Addiego F., Toniazzo V., Dubois P., Ruch D.: New development on plasticized poly(lactide): Chemical grafting of citrate on PLA by reactive extrusion. European Polymer Journal, 48, 404415 (2012).

DOI: $10.1016 /$ j.eurpolymj.2011.12.001

[13] Yang S-L., Wu Z-H., Yang W., Yang M-B.: Thermal and mechanical properties of chemical crosslinked polylactide (PLA). Polymer Testing, 27, 957-963 (2008). DOI: $10.1016 /$ j.polymertesting.2008.08.009 
[14] Quynh T. M., Mitomo H., Nagasawa N., Wada Y., Yoshii F., Tamada M.: Properties of crosslinked polylactides (PLLA \& PDLA) by radiation and its biodegradability. European Polymer Journal, 43, 1779-1785 (2007). DOI: $10.1016 /$ j.eurpolymj.2007.03.007

[15] Scott H. G.: Cross-linking of a polyolefin with a silane. U.S. Patent 3646155 A, USA (1972).

[16] Morshedian J., Hoseinpour P. M.: Polyethylene crosslinking by two-step silane method: A review. Iranian Polymer Journal, 18, 103-128 (2009).

[17] Chen T., Wang J., Shi P., Li Q., Wu C.: Effect of hot water and water-carrying agent on the properties of silanewater crosslinked linear low density polyethylene. International Polymer Processing, 28, 180-187 (2013). DOI: $10.3139 / 217.2672$

[18] Han C., Bian J., Liu H., Han L., Wang S., Dong L., Chen S.: An investigation of the effect of silane water-crosslinking on the properties of poly(L-lactide). Polymer International, 59, 695-703 (2010).

DOI: $10.1002 /$ pi.2760
[19] Rahmat M., Ghasemi I., Karrabi M., Azizi H., Zandi M., Riahinezhad M.: Silane crosslinking of poly(lactic acid): The effect of simultaneous hydrolytic degradation. Express Polymer Letters, 9, 1133-1141 (2015). DOI: 10.3144 /expresspolymlett.2015.101

[20] Raquez J-M., Narayan R., Dubois P.: Recent advances in reactive extrusion processing of biodegradable polymer-based compositions. Macromolecular Materials and Engineering, 293, 447-470 (2008).

DOI: $10.1002 /$ mame. 200700395

[21] Tambe C., Dewesthale S., Shi X., Graiver D., Narayan R.: Silylation of non-terminal double bonds of natural oils. Silicon, 8, 87-98 (2016).

DOI: $10.1007 / \mathrm{s} 12633-015-9299-2$

[22] Avella M., Errico M. E., Immirzi B., Malinconico M., Martuscelli E., Paolillo L., Falcigno L.: Radical polymerization of poly(butyl acrylate) in the presence of poly(L-lactic acid), 1. Synthesis, characterization and properties of blends. Die Angewandte Makromolekulare Chemie, 246, 49-63 (1997). DOI: 10.1002/apmc.1997.052460104 